\title{
Erratum to: Rapid quantification of alpha emitters in low- and intermediate-level dry radioactive waste
}

\author{
Tae-Hong Park ${ }^{1} \cdot$ Eul-Hye Jeon ${ }^{1} \cdot$ Yong Sul Choi $^{1} \cdot$ Jong-Ho Park $^{1}$. \\ Hong Joo Ahn ${ }^{1}$ Yong Jun Park ${ }^{1}$
}

Published online: 5 January 2016

(C) Akadémiai Kiadó, Budapest, Hungary 2015

\section{Erratum to: J Radioanal Nucl Chem DOI 10.1007/s10967-015-4177-3}

In the original publication, the units of the activities in Tables 2 and 3 are incorrect and they need to be corrected to $\mathrm{Bq} / \mathrm{kg}$. In addition, 'Activity of ${ }^{241} \mathrm{Am}$ ' in the right column in Table 3 needs to be corrected to 'Activity'. Therefore, corrected Tables 2 and 3 are given below:
Table 2 Activity measurement of $\mathrm{Am}$ and $\mathrm{Cm}$ isotopes in the radioactive dry waste sample using alpha spectrometry

\begin{tabular}{lllll}
\hline Sample \# & \multicolumn{2}{l}{ Activity $(\mathrm{Bq} / \mathrm{kg})^{\mathrm{a}}$} & $\begin{array}{l}{ }^{243} \mathrm{Am} \\
\text { recovery }(\%)\end{array}$ \\
\cline { 2 - 4 } & ${ }^{241} \mathrm{Am}$ & ${ }^{242} \mathrm{Cm}$ & ${ }^{244} \mathrm{Cm}$ & \\
\hline 1 & $198 \pm 6$ & $0.98 \pm 0.27$ & $55.4 \pm 2.5$ & 65.4 \\
2 & $203 \pm 7$ & $1.29 \pm 0.32$ & $55.6 \pm 2.6$ & 76.8 \\
3 & $205 \pm 7$ & $0.86 \pm 0.29$ & $61.9 \pm 3.0$ & 58.2 \\
4 & $208 \pm 7$ & $1.25 \pm 0.32$ & $58.4 \pm 2.7$ & 66.8 \\
5 & $194 \pm 6$ & $1.05 \pm 0.28$ & $57.4 \pm 2.6$ & 73.9 \\
Mean $(\sigma)$ & 202 & 1.09 & 57.8 & $68.2(6.6)$ \\
\hline
\end{tabular}

${ }^{a}$ Activity \pm combined uncertainty $(k=1)$

The online version of the original article can be found under doi:10.1007/s10967-015-4177-3.

Tae-Hong Park

parktae@kaeri.re.kr

1 Nuclear Chemistry Research Division, Korea Atomic Energy

Research Institute, 989-111 Daedeok-daero, Yuseong-gu,

Daejeon 305-353, Korea 
Table 3 Activity measurement of $\mathrm{Pu}$ isotopes in the radioactive dry waste sample using alpha spectrometry and TIMS

\begin{tabular}{|c|c|c|c|c|c|c|}
\hline \multirow[t]{3}{*}{ Sample \# } & \multicolumn{3}{|c|}{ Alpha spectrometry } & \multirow{2}{*}{\multicolumn{3}{|c|}{$\frac{\text { TIMS }}{\text { Activity }(\mathrm{Bq} / \mathrm{kg})^{\mathrm{a}}}$}} \\
\hline & \multicolumn{2}{|c|}{$\overline{\text { Activity }(\mathrm{Bq} / \mathrm{kg})^{\mathrm{a}}}$} & \multirow[t]{2}{*}{${ }^{242} \mathrm{Pu}$ recovery $(\%)$} & & & \\
\hline & ${ }^{238} \mathrm{Pu}$ & ${ }^{239,240} \mathrm{Pu}$ & & ${ }^{239} \mathrm{Pu}$ & ${ }^{240} \mathrm{Pu}$ & ${ }^{241} \mathrm{Pu}$ \\
\hline 1 & $58.6 \pm 2.7$ & $181 \pm 6$ & 71.3 & $164 \pm 1$ & $31.8 \pm 0.3$ & $2360 \pm 36$ \\
\hline 2 & $65.6 \pm 3.4$ & $204 \pm 8$ & 85.7 & $162 \pm 1$ & $33.1 \pm 0.3$ & $2450 \pm 30$ \\
\hline 3 & $67.9 \pm 2.8$ & $180 \pm 6$ & 76.0 & & & \\
\hline 4 & $67.6 \pm 3.4$ & $190 \pm 8$ & 52.6 & & & \\
\hline 5 & $71.4 \pm 3.5$ & $197 \pm 8$ & 50.2 & & & \\
\hline Mean $(\sigma)$ & 66.2 & 190 & $67.2(13.7)$ & 163 & 32.5 & 2405 \\
\hline
\end{tabular}

${ }^{\text {a }}$ Activity \pm combined uncertainty $(k=1)$ 